\title{
A Germanium-Based, Coded Aperture Imager
}

K.P. Ziock, N. Madden, E. Hull, W. Craig, T. Lavietes, C. Cork

This article was submitted to 2001 Nuclear Science Symposium and Medical Imaging Conference, San Diego, CA, November 4-10, 2001

\section{October 31, 2001}




\section{DISCLAIMER}

This document was prepared as an account of work sponsored by an agency of the United States Government. Neither the United States Government nor the University of California nor any of their employees, makes any warranty, express or implied, or assumes any legal liability or responsibility for the accuracy, completeness, or usefulness of any information, apparatus, product, or process disclosed, or represents that its use would not infringe privately owned rights. Reference herein to any specific commercial product, process, or service by trade name, trademark, manufacturer, or otherwise, does not necessarily constitute or imply its endorsement, recommendation, or favoring by the United States Government or the University of California. The views and opinions of authors expressed herein do not necessarily state or reflect those of the United States Government or the University of California, and shall not be used for advertising or product endorsement purposes.

This is a preprint of a paper intended for publication in a journal or proceedings. Since changes may be made before publication, this preprint is made available with the understanding that it will not be cited or reproduced without the permission of the author.

This report has been reproduced directly from the best available copy.

Available electronically at http://www.doe.gov/bridge

Available for a processing fee to U.S. Department of Energy and its contractors in paper from

U.S. Department of Energy

Office of Scientific and Technical Information

P.O. Box 62

Oak Ridge, TN 37831-0062

Telephone: (865) 576-8401

Facsimile: (865) 576-5728

E-mail: reports@adonis.osti.gov

Available for the sale to the public from

U.S. Department of Commerce

National Technical Information Service

5285 Port Royal Road

Springfield, VA 22161

Telephone: (800) 553-6847

Facsimile: (703) 605-6900

E-mail: orders@ntis.fedworld.gov

Online ordering: http://www.ntis.gov/ordering.htm

OR

Lawrence Livermore National Laboratory

Technical Information Department's Digital Library

http://www.llnl.gov/tid/Library.html 


\title{
A Germanium-Based, Coded Aperture Imager
}

\author{
Klaus P. Ziock, Norm Madden, Ethan Hull, William Craig, Tony Lavietes, Chris Cork
}

We describe a coded-aperture based, gamma-ray imager that uses a unique hybrid germanium detector system. A planar, germanium strip detector, eleven millimeters thick is followed by a coaxial detector. The $19 \mathrm{x}$ 19 strip detector ( $2 \mathrm{~mm}$ pitch) is used to determine the location and energy of low energy events. The location of high energy evcat are determined from the location of the Compton scatter in the planar detector and the energy is determined from the sum of the coaxial and planar energies. With this geometry, we obtain useful quantum efficiency in a position-sensitive mode out to $\mathbf{5 0 0}$ $\mathrm{keV}$. The detector is used with a $19 \times 17$ URA coded aperture to obtain spectrally resolved images in the gammaray band. We discuss the performance of the planar detector, the hybrid system and present images taken of laboratory sources.

\section{INTRODUCTION}

Over the past decade, a new class of gamma-ray instrumentation has been developed which builds on the wellestablished practice of using gamma-ray detectors for the control of fissile material. These instruments take advantage of the directional nature of the radiation to generate images of the location and distribution of the materials.[1-3] Some of these instruments also possess energy resolution, allowing them to further enhance the information available to the operator. Energy resolution allows both isotope specific images (showing the location of specific nuclear species present in the field of view) as well as the ability to improve the signal to noise ratio in a given image. However, current spectrally resolving gamma-ray imagers are based on the modest resolving power of alkali-halide based detectors. The use of germanium detectors with their high spectral resolution can provide even greater benefit to gamma-ray imagers, than it provides to classic, non-imaging detectors.

Expected signal-to-noise ratio enhancements of a $\mathrm{Ge}$ based imager over the current generation alkali-halide systems is shown graphically in Fig. 1 where we present the results of an MCNP simulation.[4] In the simulation a self-opaque, circular, ${ }^{239} \mathrm{Pu}$ piece $6 \mathrm{~cm}$ in diameter is placed behind a 0.5 $\mathrm{cm}$ thick, $17 \mathrm{~cm}$ diameter enriched uranium piece, both placed inside of a $24 \mathrm{~cm}$ cubic box and surrounded with organic packing material. The whole assembly is placed behind

Manuscript received November 10,2001 . Portions of this work were performed under the auspices of the U.S. Department of Energy by Lawrence Livermore National Laboratory under contract no. W-7405-Eng-48

K.P. Ziock, W. Craig and T. Lavietes are with Lawrence Livermore National Laboratory, Livermore, CA 94551 USA (telephone: 925-4234082, e-mail: ziock1@llnl.gov.)

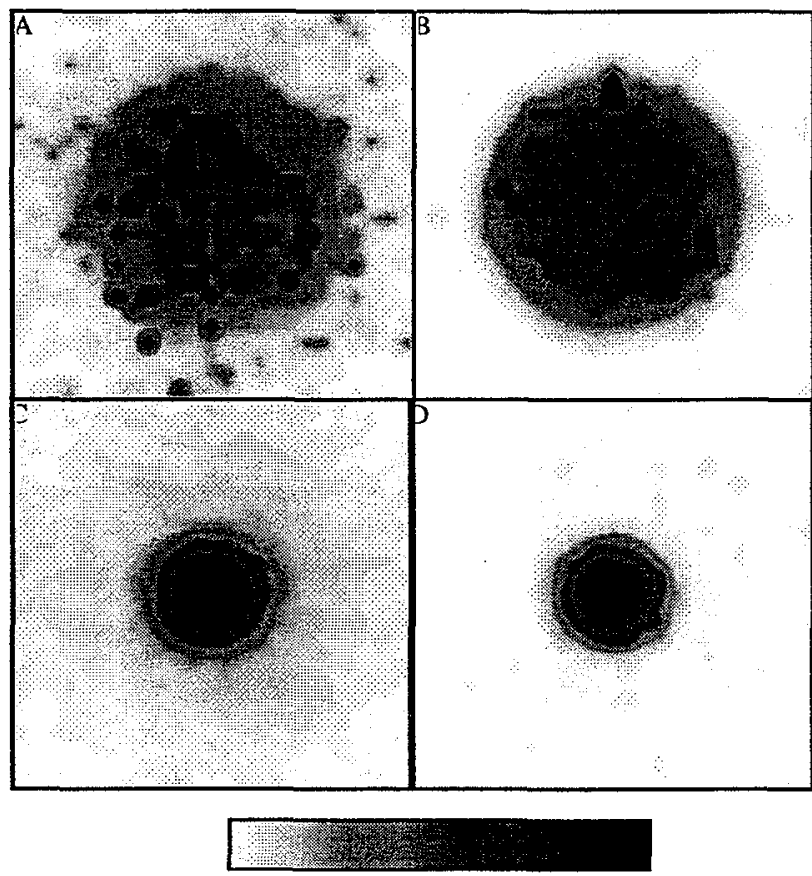

Figure 1. Simulated images of the sources described in the text. A and $B$ are of the large uranium piece $(186 \mathrm{keV}), \mathrm{C}$ and $\mathrm{D}$ are of the plutonium piece $(375 \mathrm{keV})$ which is behind the uranium. The images on the left are based on NaI energy resolution while the images on the right use germanium energy resolution. The $\mathrm{NaI}$ images have 1.5 times the integration time of the germanium images. (False color intensity is maximum for black.)

a concrete wall $10 \mathrm{~cm}$ thick. The superior performance of the Ge system, with its improved energy resolution, is evident in the simulations.

\section{DESCRIPTION OF INSTRUMENT}

The complete instrument is shown in Figure 2. The imager uses a coded aperture, indirect, imaging technique[4] coupled to a unique two-part, position-sensitive, germanium detector. The instrument takes advantage of the fact that Compton scattering is the predominate interaction mechanism of gamma-rays at high energies in germanium. An 11 $\mathrm{mm}$ thick, cross-strip, planar detector is followed by a commercial coaxial detector. At low energies the planar detector solely determines the energy and location of an event. At higher energies, the gamma-ray will likely scatter and we use the cross-strip front detector to determine the location of the scatter and then use the coaxial detector to absorb the scattered radiation. The sum of the energies in the two detectors determines the energy of the event.

N. Madden, E. Hull, and C. Cork are with Lawrence Berkeley National Laboratory, Berekeley, CA 94720 USA. 


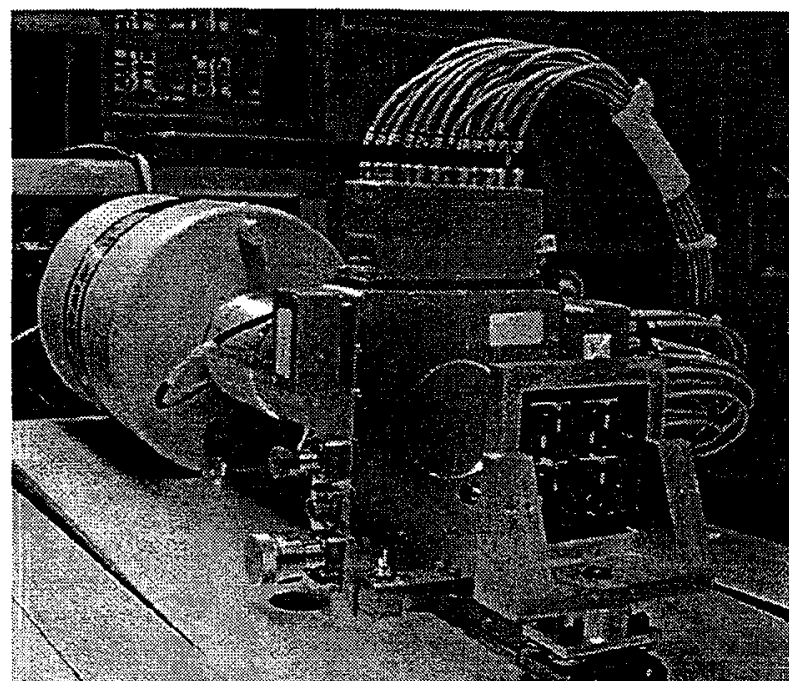

Figure 2. Photograph of the hybrid detector and the coded aperture use to acquire the images shown blow. The planar detector is in the front cryostat and is shown in Fig. 3 with the cover removed.

\section{A. Planar Strip Detector}

Details of the planar detector construction can be found in [5] and are summarized briefly below. The patterned crystal is shown mounted in the cryostat in Figure 3. It was fabricated from a $7 \mathrm{~cm}$ diameter, $1.1 \mathrm{~cm}$ thick germanium wafer using an amorphous-germanium, blocking-contact, technique.[5] The detector crystal has a net impurity concentration of approximately $2 \times 10^{9} / \mathrm{cm}^{3}$ n-type, which is sufficiently low to provide uniform electric field distribution and full depletion

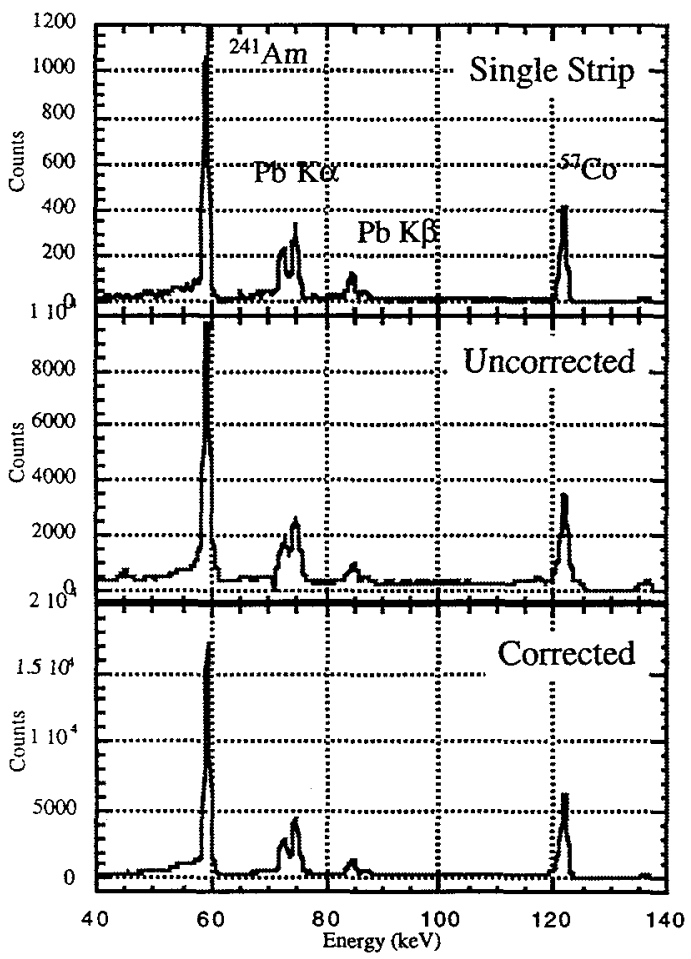

Figure 4. Energy resolution of a single strip (top) and of the full planar detector. The uncorrected full face resolution (middle) is the same at $59.5 \mathrm{keV}$ for both the single strip and corrected cases (1.3 keV).

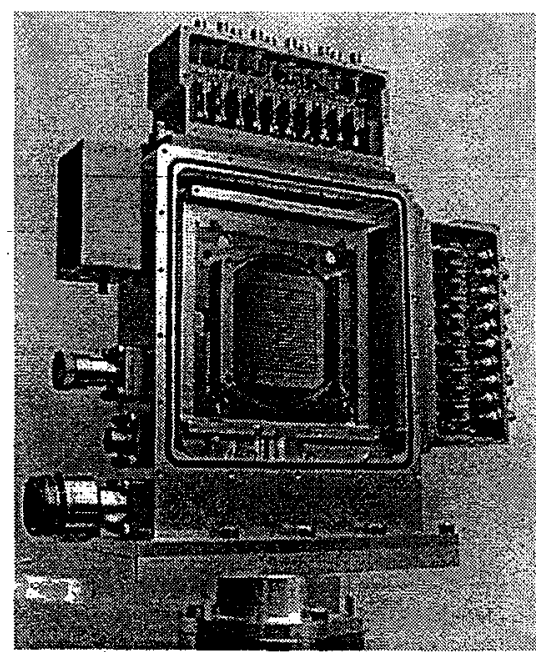

Figure 3. Picture of strip detector mounted in the cryostat. The strips on this side of the device run horizontally.

without excessively high bias voltage. The electrodes are deposited on the surface of the amorphous Ge using aluminum vapor deposition. To ensure co-registration between the crossed strips and the electrical contact in the cryostat, the entire fabrication process is conducted in a holder also used to mount the finished detector in the cryostat. All alignments are made with respect to the holder, including that of the shadow masks used in the Al deposition process. With this technique, precision structures can be manufactured reliably. The detector has $19 \times 19$ strips that are deposited on a two millimeter pitch with $500 \mu \mathrm{m}$ spacing between the strips. Guard rings $4 \mathrm{~mm}(7 \mathrm{~mm})$ wide surround the strips on the $\mathrm{AC}(\mathrm{DC})$ coupled sides of the detector.

\section{B. Electronics}

Custom electronics were constructed to handle the signals from the planar system. A small hybrid preamplifier[6] (visible on the top and right sides of Figure 3) mounted on the cryostat at room temperature extracts the charge signals from the illuminated strips on the two sides of the detector. These signals are recognized with fast discriminators creating the $X$ and $\mathrm{Y}$-axis photon interaction addresses. The $\mathrm{X}$-axis electronics also has slower spectroscopic pulse shaping filter/amplifiers, peak detector/stretchers, and an analog multiplexer. An overall local logic card selects the analog signal that passes the multiplexer for processing by the digital circuitry. The digital electronics checks for the presence of a signal on both $\mathrm{X}$ and $\mathrm{Y}$ strips and (if enabled) the coaxial detector. (The signals for the latter are processed using standard NIM electronics.) The digital electronics convert the analog signals to a digital number (energy) and adds an $\mathrm{X}$ and $\mathrm{Y}$ address (interaction position), for processing by the data acquisition computer. A programmable gate array parses the data and stores it in local memory. An embedded processor is used to pass the information to the host computer for archival and display. 


\section{PERFORMANCE}

\section{A. Planar Detector}

\section{1) Energy Resolution}

The first tests on the system were conducted using low energy sources to check the response of the planar detector only. The energy resolution of a single strip at $122 \mathrm{keV}$ was found to be FWHM $=1.30 \mathrm{keV}$ for the DC coupled channel and FWHM $=1.41 \mathrm{keV}$ for the AC coupled channel. These spectra were measured with $T_{P}=2 \mu \mathrm{s}$ and a $90 \mu \mathrm{Ci}{ }^{57} \mathrm{Co}$ source $12.1 \mathrm{~cm}$ from the DC side of the detector. A bias of $400 \mathrm{~V}$ was used to fully deplete the detector. The resolution is $\sim 6 \%$ worse for the DC side if the source is positioned over the AC side of the detector. This is presumably due to trapping of the electrons that are collected by this electrode.

The full-face energy resolution of the strip detector is shown in Figure 4 and is found to be $1.3 \mathrm{keV}$ at $59.5 \mathrm{keV}$. To achieve this required matching the electronic gains of the individual strips. This was performed in two steps. The approximate gains were set by changing a variable gain resistor to match the line spectra from an ${ }^{241} \mathrm{Am}$ source on an oscilloscope. A full-face illumination of the detector with both a ${ }^{241} \mathrm{Am}$ and a ${ }^{57} \mathrm{Co}$ source was then obtained. This data was used to calculate residual gain and offset differences from the different strips, and these values are used by the software to correct the data on an event-by-event basis.

\section{2) Position Resolution}

The position resolution is determined by the $2 \mathrm{~mm}$ pixel size. It can be seen in Figure 4, where we project the shadow of a key onto the device. The dark regions at the bottom and top of the detector indicate that relatively small numbers of events are recorded in the end strips. This can be attributed to
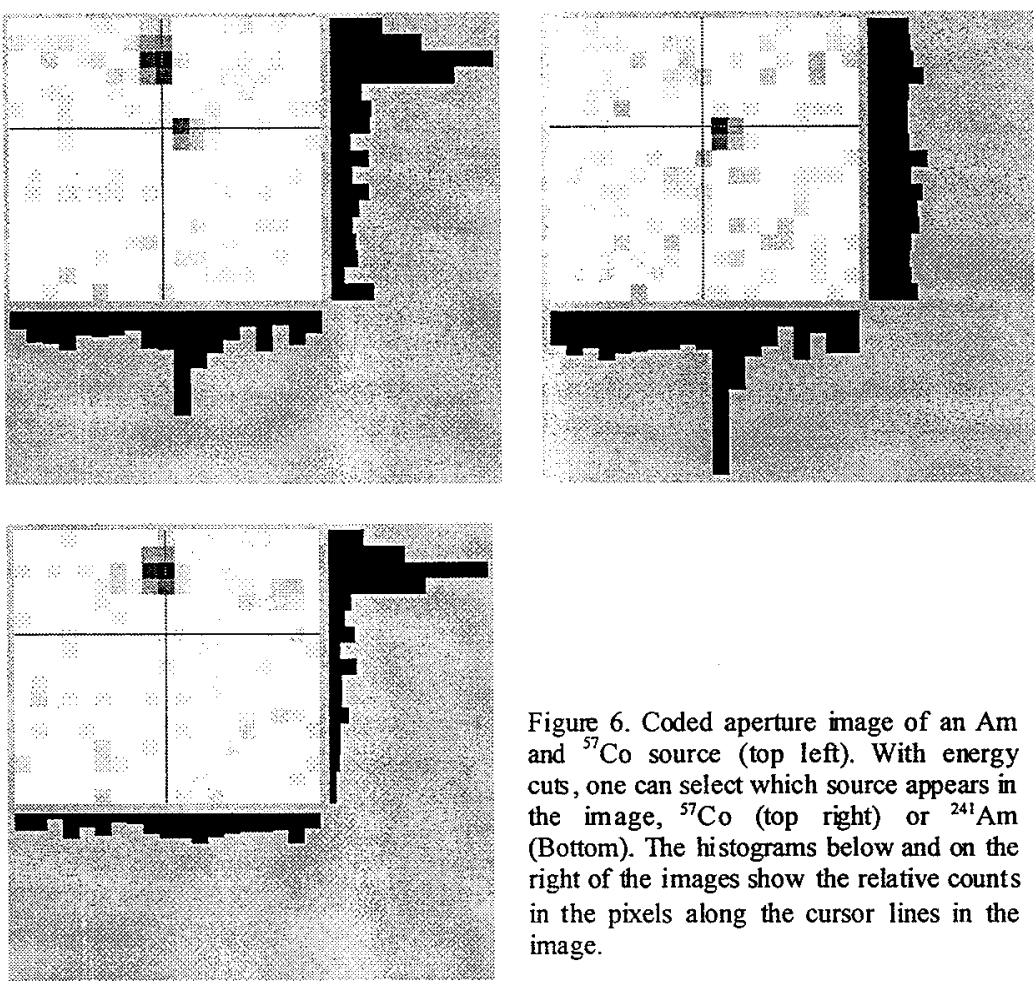

Figure 6. Coded aperture image of an Am and ${ }^{57} \mathrm{Co}$ source (top left). With energy cuts, one can select which source appears in the image, ${ }^{57} \mathrm{Co}$ (top right) or ${ }^{241} \mathrm{Am}$ (Bottom). The histograms below and on the right of the images show the relative counts in the pixels along the cursor lines in the image.

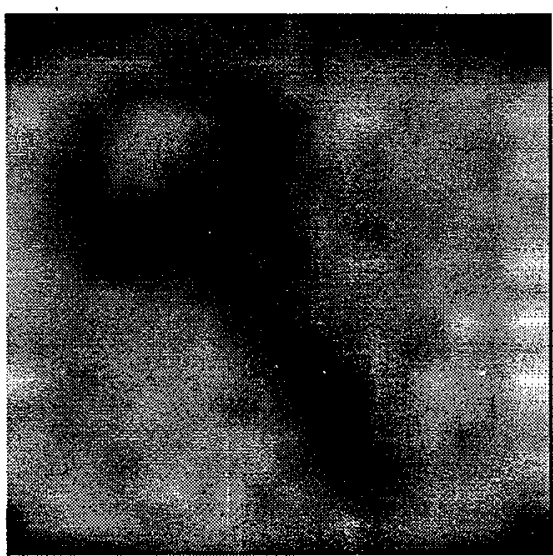

Figure 5. Shadow of a key projected on the detector by an ${ }^{241} \mathrm{Am}$ source. The image has been smoothed to remove pixellation effects.

field lines terminating on the guard rings and is partially due to the low applied bias voltage.

\section{3) Imaging Performance}

A $19 \times 17$ coded aperture was used in front of the detector to obtain a coded aperture image. The data is taken in two steps using equal mask and anti-mask integrations to remove background variations in count versus position at the detector. [6] The results are shown in Figure 6 where both an ${ }^{241} \mathrm{Am}$ and ${ }^{57} \mathrm{Co}$ source are visible in the image.

We have instituted a novel, "full-deconvolution" of the data, in which we generate a separate image for each of the 1024 channels of energy data. With this technique one can rapidly make cuts in both energy and position to see the effect on the image. Of particular value is the fact that the energy spectrum from a single (or range) of pixels can be rapidly determined. This is shown in Figure 7 where the energy spectra from the indicated pixels are used to reveal the identity of the sources.

\section{B. Hybrid Detector}

The inclusion of a coaxial detector behind the planar, position-sensitive detector is meant to expand the useful energy range of the resulting imager. At $100 \mathrm{keV}$ the mean free path of a gamma-ray in $\mathrm{Ge}$ is $\sim 3.5 \mathrm{~mm}$. This means that we have both a high probability of capturing the gamma-ray and containing the full energy of an event within a single $2 \mathrm{~mm}$ square by $11 \mathrm{~mm}$ long pixel. The full deposition of all energy is important since the ratio of the photoelectric to incoherent Compton cross section is already $1: 3$; meaning that it is likely multiple interactions will occur within the pixel. By the time we reach $300 \mathrm{keV}$ the mean free path is $1.6 \mathrm{~cm}$ and the ratio of photoelectric to incoherent Compton cross section is $1: 6.5$. Thus it is unlikely that we will stop a gamma-ray in a single pixel. To overcome this difficulty, we require events at higher energy to deposit energy in both the planar detector and the coaxial detector. Further, we look for 

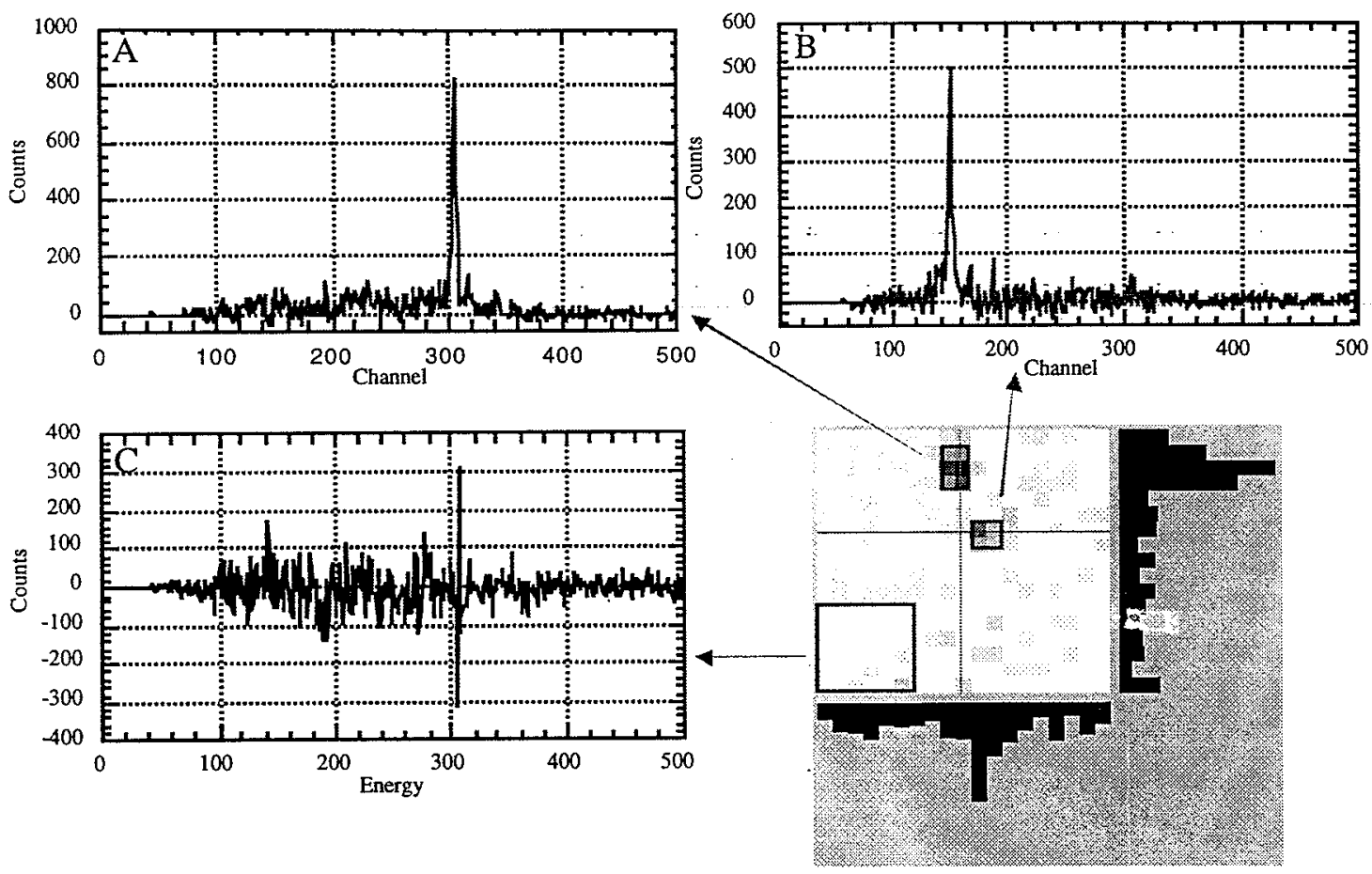

Figure 7. Early coded aperture image taken with planar detector only. The image is shown on the lower left. The energy spectra (uncalibrated) are obtained from the indicated pixels using the "full-deconvolution" technique described in the text. The spectra identify the upper source (A) as ${ }^{57} \mathrm{Co}$ and the lower source (B) as ${ }^{241} \mathrm{Am}$. The third spectrum is taken from a background region. Note that the noise in the spectra comes from the coded aperture imaging process.

events that represent a single scatter in the planar detector with full photo-absorption of the residual photon in the coaxial detector. In this way, we both increase the quantum efficiency of the detector and improve the quality of the image that is obtained.[4]

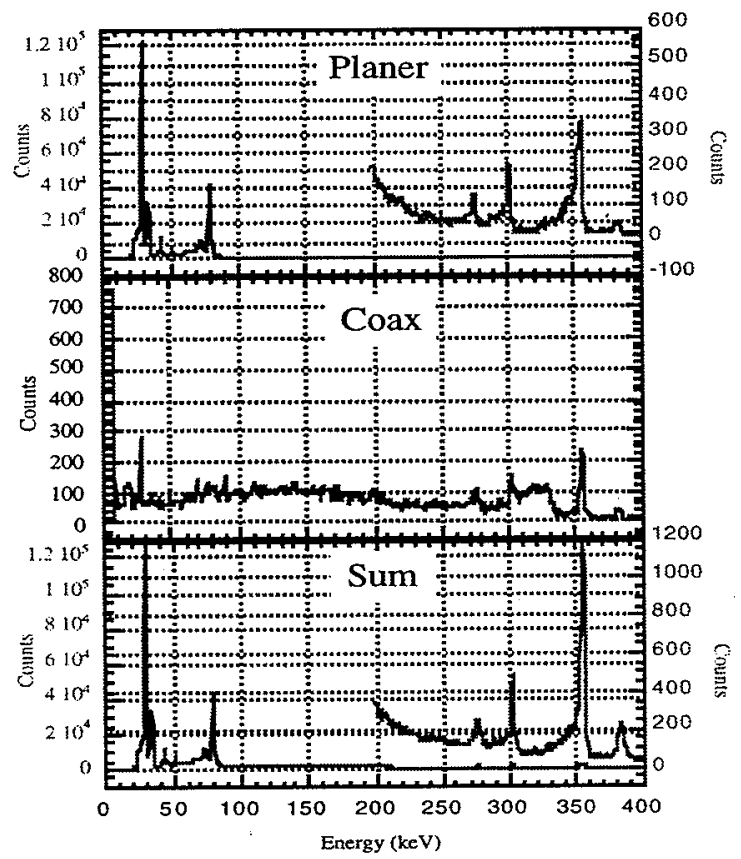

Figure 8. Hybrid detector response to flood-field illumination by $\mathrm{a}^{133} \mathrm{Ba}$ source. Note that the Coaxial detector does not show any photopeak events because the trigger conditions require some energy deposition in the planar detector. The bottom spectrum shows a four-fold increase in $356 \mathrm{keV}$ detected counts compared to the planar only data.
The improved efficiency of the combined detector is shown in Fig. 8 where we present the spectrum from a ${ }^{133} \mathrm{Ba}$ source as recorded by the instrument. The upper spectrum was obtained with just the planar detector. Not only is the quantum efficiency low, but the events in the $356 \mathrm{keV}$ peak are likely distributed across several pixels, meaning that they will produce a poor image. Note that the coincidence requirement means that there is only a small (accidental) photopeak visible in the coaxial detector. The data from the summed system shows a four-fold increase in quantum efficiency at $356 \mathrm{keV}$ between the planar only and the full system.

If we keep only events in the summed photopeak, then we see characteristic spectra in both the planar and the coaxial detector as shown in Figure 9. The spectrum from the planar detector is dominated by the low energy shallow scatter events. The spectrum from the coaxial detector is dominated by high energy events which represent the rest of the energy of the $356 \mathrm{keV}$ photons. Events to be used for image processing are those below $92 \mathrm{keV}$ (lower energy line in the Figure) in the planar detector. These events are most likely to have undergone only a single scatter in the planar detector. The position of this energy cut off is determined from the system geometry shown in Figure 10. The maximum scatter angle for a single scatter is given by the angle $\phi$ shown in the figure. The size of this angle is used in the Compton formula:

$$
\cos \theta=1+m_{e} c^{2}\left\lfloor\frac{1}{E_{0}}-\frac{1}{E_{0}-E_{r e}}\right\rfloor
$$




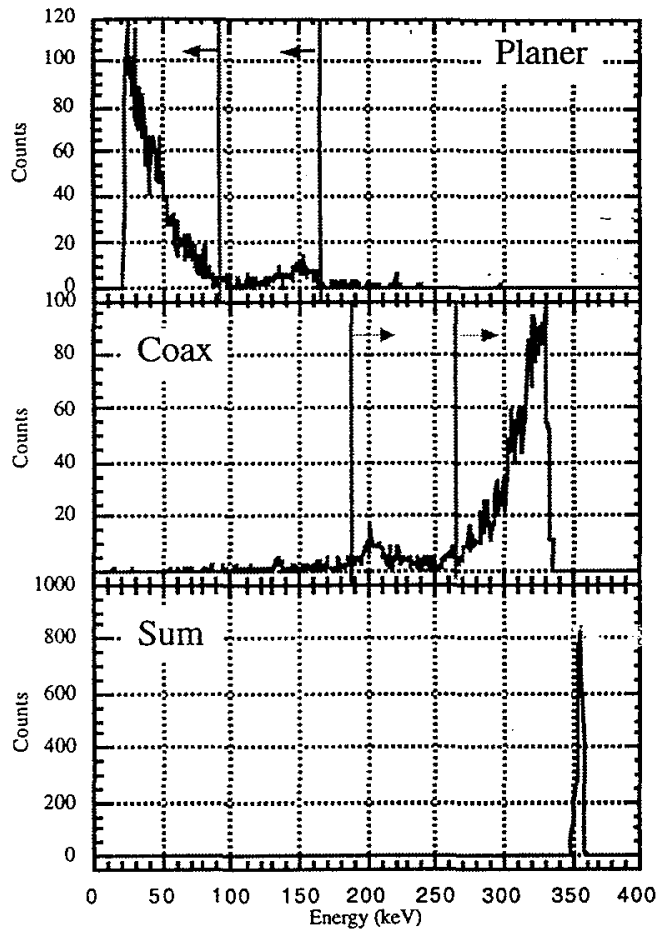

Figure 9. Energy spectra observed in the planar and coaxial detector when only coincident events summing to $356 \mathrm{keV}$ are kept. The valid events have energy $<92 \mathrm{keV}$ in the planar detector and more than 264 $\mathrm{keV}$ in the coaxial detector. The broad peak next to the dark line at higher (lower) energy in the planar (coax) detector represent backscatter events which first interact in the coaxial detector.

where theta, is the scatter angle, $\mathrm{m}_{\mathrm{e}} \mathrm{c}^{2}$ is the electron rest mass, $E_{0}$ is the incident photon energy and $E_{\mathrm{re}}$ is the energy of the recoiling photon. From this one determines the maximum recoil energy possible. With knowledge of the system geometry one can select the optimum energy cuts for each of the energy bins in the system.

\section{1) Imaging Performance}

The performance of the instrument as an imager was determined using the same $19 \times 17$ URA masks and the mask/anti-mask imaging technique used for the planar only images. In Figure 11 we show the response to the $511 \mathrm{keV}$ line from a ${ }^{22} \mathrm{Na}$ source.

\section{FUTURE PLANS}

The $19 \times 19$ strip detector was constructed primarily as a prototype to test out imager design. We are now in the final stages of assembling a larger $38 \times 38$ strip detector. A number of new design features will be incorporated in this in-

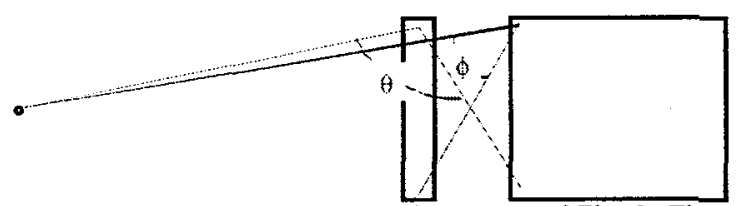

Figure 10. Geometry which explains the spectra of Fig. 9. The dark inner line represents bad events which first interact in the coaxial detector. These backscatters through a maximum angle $\phi$ before detection in the planar detector. Valid events are indicated by the light line which can have a maximum energy deposition in the planar detector determined by the angle $\theta$.
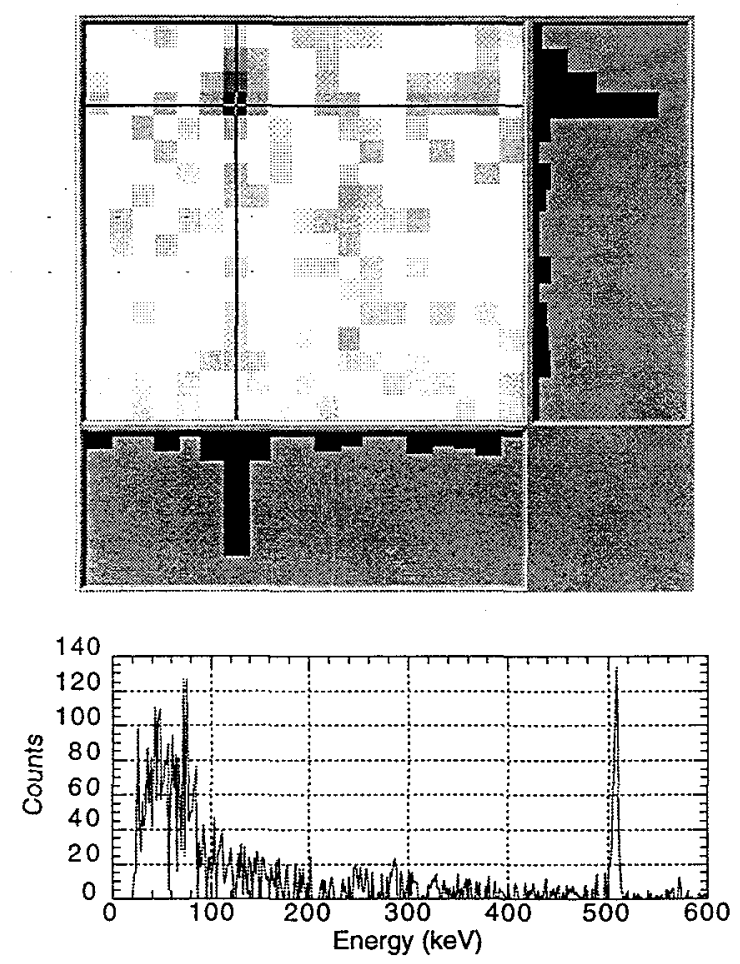

Figure 11. Image of $511 \mathrm{keV}$ point source (top) and the spectrum from the hot pixel (bottom)

strument. The larger area of this detector will increase the sensitivity of the imager. It will also allow for a proper oversampling of the coded aperture pattern. Our goal is to maintain the same number of resolution elements in the picture. To achieve this we will incorporate a $19 \times 19$ MURA pattern[7] which has the advantage that it is its own anti-mask upon a 90 degree rotation. To further increase the efficiency of the unit, we will build a single cryostat to house both the planar and the coaxial detector. This will allow us to bring the two into close contact that should increase the detector efficiency. Original simulations showed an optimal imaging detector QE of $8 \%$ at $400 \mathrm{keV}$ while we have measured only $6 \%$ at $356 \mathrm{keV}$. The separation between the two detectors is an obvious difference between the measured and simulated systems.

\section{ACKNOWLEDGMENT}

We gratefully acknowledge the cryostat design work performed at LLNL by Del Eckels and the work on the system housing by Eric Fought and Randy Hill.

\section{REFERENCES}

[1] K.P. Ziock, Science and Technology Review, UCRL-52000-95-10, Oct. 1995. p. 14.

[2] M. Woodring, et al. NIM, A422, 709, 1999.

[3] AIL SYSTEMS INC. 455 Commack Road, Deer Park, New York 11729-4591 USA.

[4] K.P. Ziock, et al., Proceedings of the $41^{\text {st }}$ Annual INMM Meeting, New Orleans, LA, July, 2000

[5] E.L. Hull, et al., to be published in SPIE Proceedings 4507, (SPIE, Bellingham Washington, 2001.)

[6] K.P. Ziock, C.J. Hailey, T.B. Gosnell, J.H. Lupton, F.A. Harrison, IEEE Trans. Nucl. Sci., 39, 1046, 1992.

[7] S.R. Gottesman, E.E. Fenimore, Applied Optics, 28, 4344, 1989. 